\title{
Polarized Laser Beam Interaction With Soft Tissue Matter
}

\author{
K. Rajkumar \\ Associate Professor SR Engineering College, Telangana, INDIA \\ V. Padmaja \\ Professor VNR VJIET Telangana, INDIA
}

\begin{abstract}
We present the investigational consequences for thinning out intensity which arises from elucidation of tissue intermediate with laser light for characterizing the dissimilar types of soft particles. The imaging of tissue akin to apparition provides the option of modeling the laser tissue communication parameters for the indicative of malignant bug and shaping the optical properties of a soft material. Methodical modeling of optical equipment with Stokes Mueller matrix generates a distinctive resolution for characterization of model material. Optics direction of polarizer-analyzer arrangement provides the methodical representation for soft matter characterization using tissue imaging. A new photo polarimetric measurement system of Mueller matrix of optical elements is developed using dual rotating wave plates. The wave plates in polarization generator and analyzer rotate continuously with a constant radio of revolution speed, and the Mueller matrix of a sample can be obtained in a few seconds.
\end{abstract}

Keywords - Tissue imaging, Mueller matrix, Soft matter;

\section{INTRODUCTION}

When polarized optical light, or any electromagnetic wave incidents on a soft tissue material, it is captivated, transmitted, reflected, refracted, or speckled. These exchanges are definite scrupulously via Maxwell's equations. If any intermediate is comprised of a number of soft matter tissue substances, the providence of the happening wave surrounded by the soft medium is resolute by bearing in mind the collective interactions. If a string of assumptions are ready, these comparatively intricate equations can be replaced with simpler terminology of statistical optics. Using these statistical equations, we can carve an appearance for the protection of the radiative power in the length of a given track in the soft medium. The captivated power is punctually transformed to thermal power (heat), and the speckled power is to redistribute all the way through the soft medium. This speckled energy carries the information about the soft medium, and if it is statistically quantified appropriately, it may aid characterization of the matter in the soft medium. [1] However there has been a numeral of experimental and academic study carried out concerning polarized light transmission and distribution in casual medium for applications in soft tissue imaging [2-5]. The interaction of light with matter has been shown in below figure1. In order to extract all the available information about the optical dielectric properties of matter, polarized lights inputs are necessary. The reason for this is that the polarization of light has the measurable effect of increasing the discriminating ability of light interrogation of matter. The biological matters and other random inhomogeneous media render imaging difficult due to the random multiple scattering of light.
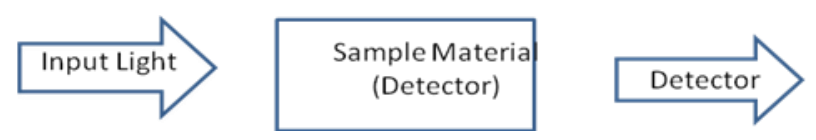
Fig 1:-Schematic model of Soft Matter interaction with
light.

After the contraption of laser, numerous researches were performed investigating potential interaction on soft matter (tissue) by means of all types of lasers and biological tissue properties. Before focusing on the interaction of light with the soft matter(Tissue)interactions, it is important to study the optical properties of soft materials( tissues). The importance is due to the dependency of light penetration inside soft material on laser parameters and optical properties of soft materials (tissues) [6]. Therefore, each biological tissue has individual impulse response functions when irradiated by laser light. This impulse reaction is thorny and many groups around the world work in this field. This logical focus is called medical optics and has important role in laser surgery, optical imaging, and photodynamic therapy [7]. Each interaction of optical light with different polarized states mechanism with the soft material will be carefully observed. These observations including typical experimental data and/or histology of tissue samples after laser exposure. In this work we will talk about basic phenomena occurring when biological tissue is irradiated by laser light. In principle, four phenomena exist which govern on undistributed propagation of light in tissue: reflection, scattering, absorption and fluorescence. Reflection can be studied by Fresnel's law [8]. Most part of laser energy penetrates into biological tissue. The infiltration of laser light in biological tissue depends on optical properties of biological tissue, such as index of refraction, scattering and anisotropic factor, and also the absorption of laser light in tissue. These optical properties resolve the apparatus of laser-tissue interaction in that special case. So, first, we study the optical properties of biological tissue, followed by the revision of the effect of these properties on infiltration of laser light. Mainly, the deeds of laser in its interface with tissue can be classified by its wavelength. Today, there are several lasers with wavelengths between x-ray and infra-red (IR). Famous lasers 
like $\mathrm{CO} 2$, neodymium-doped yttrium aluminium garnet $(\mathrm{Nd}$ : YAG), fiber laser, and dye lasers are frequently applied in medicine. The most important difference that can be used to classify lasers is their wavelength, because the molecular absorption depends on this important parameter. For example, the $200 \mathrm{~nm}$ laser light is absorbed only slightly by viruses; more is seen by bacteria cells, and an entire absorption by mammalian cells.. Oxygen consumption is activated by illuminating with light at $365 \mathrm{~nm}$. It is believed that mitochondria are the absorber of $630 \mathrm{~nm}$ [9]. Therefore, in medical optics, the wavelength of laser has an important role due to the absorption peaks of bio-molecules and tissues.

\section{MEDICAL OPTICS}

400-1100 nm spectral region is called optical window. Most biological tissues are classified by strong optical scattering in this region. So, they are referred to as turbid media (Figure2). The laser light can be simultaneously assumed as electromagnetic wave and particle beam of photon or of lasers diffuse and attenuate in biological tissues.

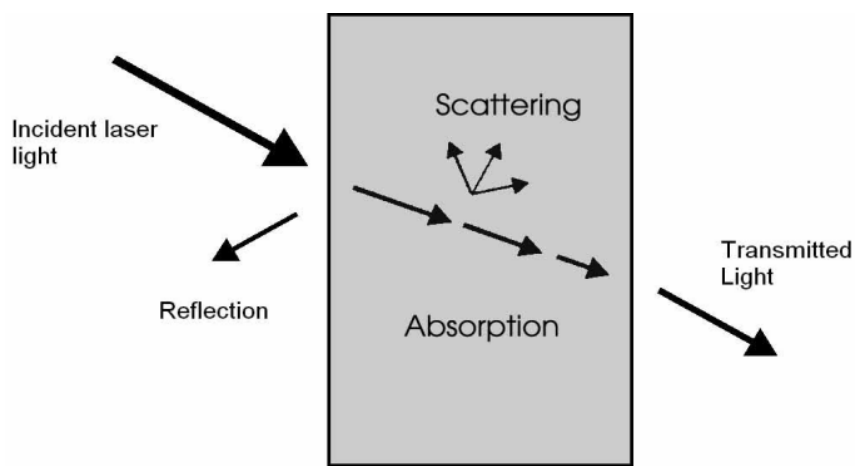

Fig 2:-Schematic of scattering in turbid media.

Scattering of laser light by cells, nuclei, mitochondria, lysosome, macromolecules, membranes and other components, results in diffusion of light in biological tissues. Optical scattering originates from light interaction with biological structures ranging from cell membranes to whole cells. The size of this scattereres varies from $10 \mathrm{~nm}$ to 10 microns. These scattereres are distributed randomly in biological tissue; photons usually come across numerous scattering actions. The dispersal coefficient $\sigma$ is defined as the chance of photon dispersal in a medium per unit path length. The dispersal signify free path (spatial interval between two adjacent scatterings) is the reciprocal of scattering coefficient $\sigma$. The scattering coefficient of biological tissues depends on the wavelength of laser, e. g. the scattering coefficient of liver for lambda of 515, 800 and $1064 \mathrm{~nm}$ is 285,200 and $150 \mathrm{~cm}-$ 1 , respectively. The scattering mean free path of liver for these wavelengths

is 35, 50 and 66 microns, which shows that the spatial interval between two scatterings increases for longer wavelengths. In most biological tissues, the majority of scattered light travels in forward direction. So, these biological tissues have anisotropy which is convenient to define a phase function $p$ $(\theta)$ of a photon to be scattered by an angle $\theta$. If $p(\theta)$ is a constant and not dependent on $\theta$, the media is called isotropic, otherwise, it is called anisotropic medium. Experiments show the mean value of phase function $g$ is a number between- 1 and +1 . Where $g=1$ denotes purely forward scattering and $g=-1$ purely backward scattering, and $g=0$ isotropic scattering. Most biological tissues have $g>0.7$ (10).

\section{METHODS AND MATERIALS}

The polarization of light is well described either via Jones or Mueller matrix algebra although the latter is preferred when dealing with depolarizing interactions as engendered by multiply scattering media. Using the Mueller matrix approach, the intensity and polarization of a light beam are represented by a 4-element Stokes vector, S. The first element I represents the intensity of the light beam, the second element Q represents the linear polarization at $0_{-}$and $90_{-}$, the third element $U$ represents the linear polarization at $45_{-}$and 135_, and the fourth element $\mathrm{V}$ represents the circular polarization. The effects of the medium under investigation are applied to this vector with a 4 _ 4 Mueller matrix M. The Stokes-Mueller description of polarized-light propagation and scattering has experienced recent popularity in a wide variety of applications. In view of the increasing number of experimental measurements of a Mueller matrix (denoted hereafter as M), physical reliability constraints on the structure of $\mathrm{M}$ become more important.

Indeed, such constraints can be used in the calibration of polarimetric instruments, the estimation of experimental errors, and the testing of computational procedures. Solutions to many problems of polarization optics sensitively depend on the relative strength of the unpolarized component, i. e., the output degree of polarization, that is introduced by the corresponding Mueller matrices. This is particularly true for various optimal reception problems, e. g., what polarization should be chosen for the best when optical light is incident with the soft tissue matter i. e. tissue. Many more constraints on Mueller matrix elements have been recorded. However, we shall not attempt to list or even to discuss them further here. The reason for this is that they may be largely irrelevant when one is making measurements with real optical systems. The measured Mueller matrices are a mixture of pure (no depolarizing) states, depolarization, and certainly noise (optical and electronic). Is the magnitude of a particular Mueller matrix element due to diattenuation or retardance or is it really noise, or is it a mixture? If it is a mixture, what are the proportions? It is the responsibility of the experimenter to reduce noise sources as much as possible, determine the physical reliability of his Mueller matrices, and if they are not physically realizable, find the closest physically realizable Mueller matrices. A method of finding the closest physically realizable Mueller matrix and a method of decomposing no depolarizing and depolarizing

\section{EXPERIMENTAL PROCEDURE}

The soft matter tissue sample was illuminated with laser of $10 \mathrm{~mW}$ power and $556 \mathrm{~nm}$ wavelength with PSG. The collection optics is kept in line from the input beam direction throughout the experiment to acquire the intensity images in 
transmission mode, and the intensity information is gathered using a CCD in the PSA system and the images are stored in JPEG format. As shown in Figure 3.

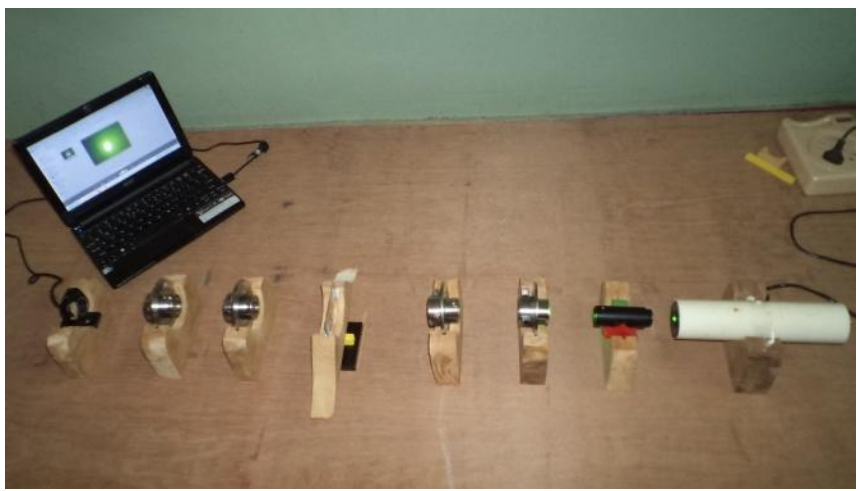

Fig. 3. Experimental setup

The samples considered here are pathology soft material tissues. The specimens selected for is a soft material tissue processing is sent in $10 \%$ formalin for proper fixation of the tissue. Unfixed and autolyzed tissues cannot be processed. The fixed specimen is grossed accordingly and specific sites for soft material tissue processing have to be identified. Then using a scalpel blade the concerned part of the specimen is cut into even square shaped bits and taken in to capsules. These capsules are then taken through different concentrations of alcohol for dehydration and hardening of the soft material tissue. It is inserted into containers with molten wax for impregnation of the wax into the tissues. This is to harden the tissue to prepare blocks which are later cut. The impregnated soft material tissues are then blocked with molten wax so that the cut surface of the breast tissue is properly aligned and then allowed to harden. These blocks are mounted onto a micro tome which cuts the breast tissue in the blocks in to 3-4 microns thick sections $[11,12]$. These sections are taken on to slides and stained with Hematoxylin and Eosin stains and is as shown in Figure4.

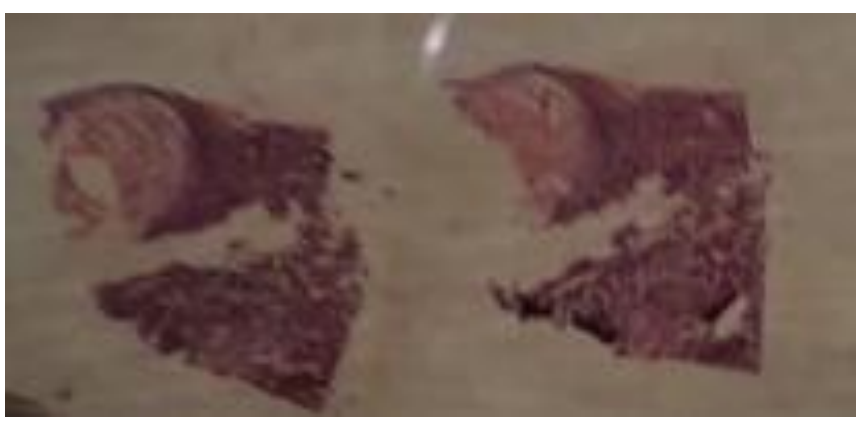

Fig. 4. Soft Tissue sample used for experiment

By varying the optical elements in the PSG and PSA, 49 different intensity images were recorded.

49 intensity images with various orientations of Polarizer and Analyzer are necessary to obtain the 16 elements of Mueller matrix images. The 49 intensity images obtained are named as follows

\begin{tabular}{|l|l|l|l|l|l|l|}
\hline $\mathrm{I}_{\mathrm{OO}}$ & $\mathrm{I}_{\mathrm{OH}}$ & $\mathrm{I}_{\mathrm{OV}}$ & $\mathrm{I}_{\mathrm{OP}}$ & $\mathrm{I}_{\mathrm{OM}}$ & $\mathrm{I}_{\mathrm{OR}}$ & $\mathrm{I}_{\mathrm{OL}}$ \\
\hline $\mathrm{I}_{\mathrm{HO}}$ & $\mathrm{I}_{\mathrm{HH}}$ & $\mathrm{I}_{\mathrm{HV}}$ & $\mathrm{I}_{\mathrm{HP}}$ & $\mathrm{I}_{\mathrm{HM}}$ & $\mathrm{I}_{\mathrm{HR}}$ & $\mathrm{I}_{\mathrm{HL}}$ \\
\hline $\mathrm{I}_{\mathrm{VO}}$ & $\mathrm{I}_{\mathrm{VH}}$ & $\mathrm{I}_{\mathrm{VV}}$ & $\mathrm{I}_{\mathrm{VP}}$ & $\mathrm{I}_{\mathrm{VM}}$ & $\mathrm{I}_{\mathrm{VR}}$ & $\mathrm{I}_{\mathrm{VL}}$ \\
\hline $\mathrm{I}_{\mathrm{PO}}$ & $\mathrm{I}_{\mathrm{PH}}$ & $\mathrm{I}_{\mathrm{PV}}$ & $\mathrm{I}_{\mathrm{PP}}$ & $\mathrm{I}_{\mathrm{PM}}$ & $\mathrm{I}_{\mathrm{PR}}$ & $\mathrm{I}_{\mathrm{PL}}$ \\
\hline $\mathrm{I}_{\mathrm{MO}}$ & $\mathrm{I}_{\mathrm{MH}}$ & $\mathrm{I}_{\mathrm{MV}}$ & $\mathrm{I}_{\mathrm{MP}}$ & $\mathrm{I}_{\mathrm{MM}}$ & $\mathrm{I}_{\mathrm{MR}}$ & $\mathrm{I}_{\mathrm{ML}}$ \\
\hline $\mathrm{I}_{\mathrm{RO}}$ & $\mathrm{I}_{\mathrm{RH}}$ & $\mathrm{I}_{\mathrm{RV}}$ & $\mathrm{I}_{\mathrm{RP}}$ & $\mathrm{I}_{\mathrm{RM}}$ & $\mathrm{I}_{\mathrm{RR}}$ & $\mathrm{I}_{\mathrm{RL}}$ \\
\hline $\mathrm{I}_{\mathrm{LO}}$ & $\mathrm{I}_{\mathrm{LH}}$ & $\mathrm{I}_{\mathrm{LV}}$ & $\mathrm{I}_{\mathrm{LP}}$ & $\mathrm{I}_{\mathrm{LM}}$ & $\mathrm{I}_{\mathrm{LR}}$ & $\mathrm{I}_{\mathrm{LL}}$ \\
\hline
\end{tabular}

here, the first subscript indicates input state Polarizing State Generator (PSG), and the second subscript the output state Polarizing State Analyzer(PSA).

\section{RESULTS AND DISCUSSION}

In order to detect the fine structural variations of small particles, we have developed the matrix model for characterization of material. We used polarized light produced by unique sets of polarize-retarder to make different and accurate scattering measurements. These unique settings are pre-determined following a series of experiments using the available algorithms. After that, the Sij elements recovered from the recorded intensities in a numerical inverse analysis. The output 16 Mueller matrix elements are measured by 49 polarization configurations, according to apparatus shown in Fig. 3 and analysis is made to find the Diattenuation, Retardance and Depolarization of the images that are obtained from the above experimental setup which is consisting of Polarizing State Generator and Polarizing State Generator via soft matter. The Mueller matrix element Sij, indicate the scattering pattern of an soft matter, and if they are determined from experimentally measured scattering values gies the characterizing nature of the soft material.

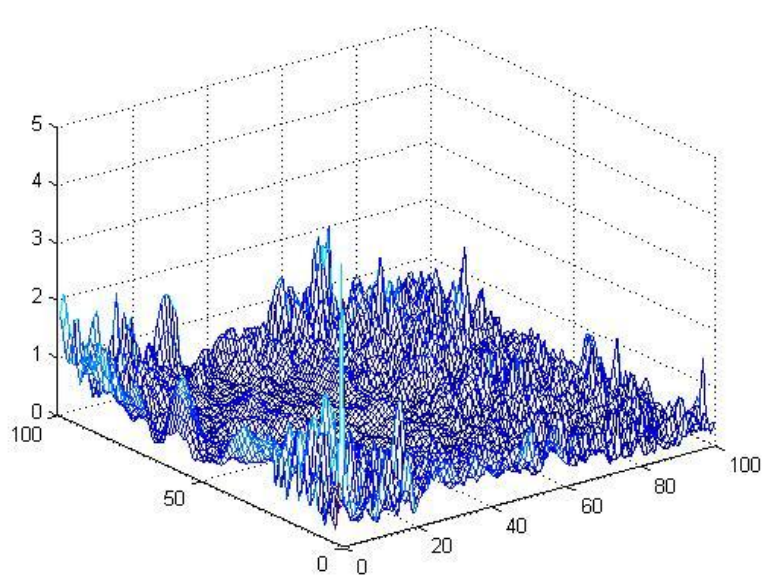

Fig. 5. Diattenuation image of Soft matter Tissue 


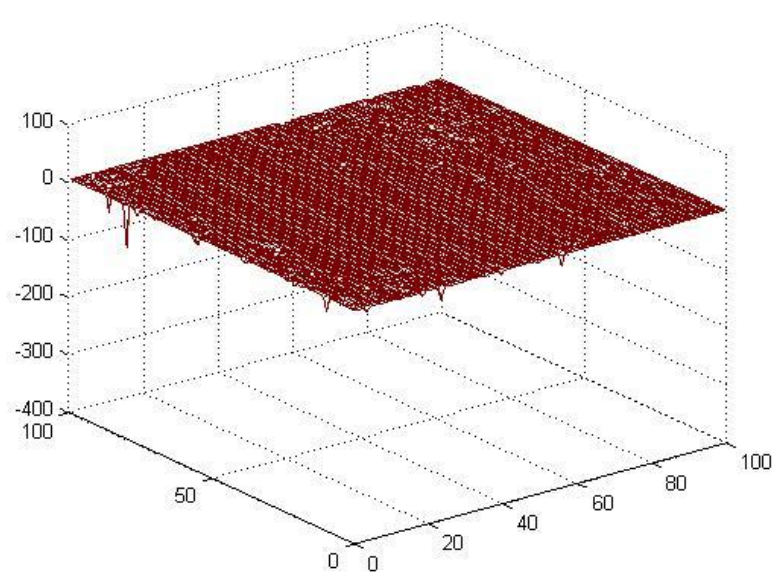

Fig. 6. Depolarization image of Soft matter Tissue

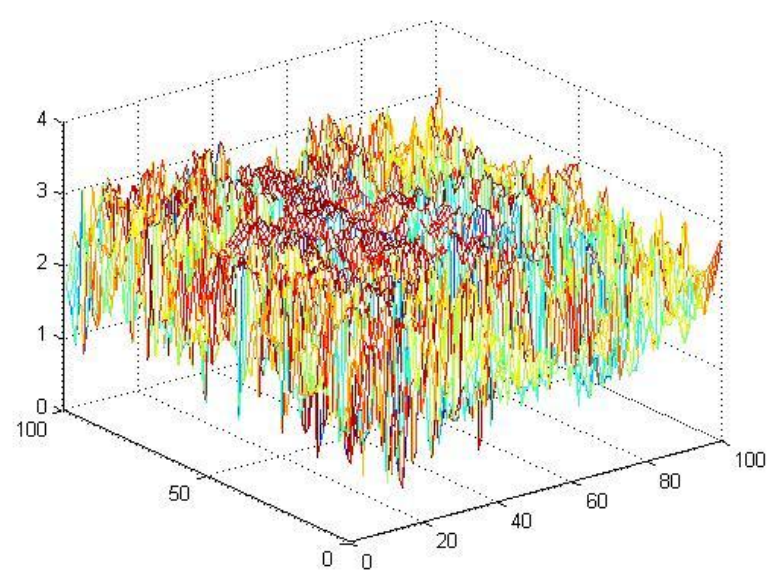

Fig. 7. Retardance image of Soft matter Tissue

From the measured Mueller matrix the Diattenuation, Retardance and Depolarization images are acquired and the images are shown in figure 4. The mean value of Diattenuation is 0.4058 , Depolarization is 0.3378 and that of Retardation is 2.2462.

\section{CONCLUSIONS}

A complete Mueller polarimeter is possible from a PSG and PSA for a soft material Tissue that is evident from the images and values obtained. The pixel by pixel processing of the images effectively resulted in understanding the polarization anisotropic changes and scattering intensity distributions present in different regions of the soft material sample.

\section{ACKNOWLEDGEMENTS:}

The authors would like to thank their parent Institutions and Organizations for their kind help, we are also grateful to Gandhi Medical College, Hyderabad for providing the tissue samples. We would like to thank the Ministry of Education
Tanzania and Dodoma University for the experimental facilities.

\section{REFERENCES:-}

[1] S. Firdous, M. Ikram, (2004) Charactrization of turbid medium through diffusely backscattered polarized light with matrix calculus-I, IEEE proceeding INCC 2004, 115-23

[2] J. M. Schmitt, A. H. Gandjbakhche, and R. F. Bonner, (1992) Use of polarized light to discriminate short-path photons in a mutiply scattering medium, Appl. Opt. 31, 6535-46.

[3] A. H. Hielscher, J. R. Mourant, and I. J. Bigio, (1997) Influence of particle size and concentration on the diffuse backscattering of polarized light from tissue phantoms and biological cell suspensions, Appl. Opt. 36, 125-35.

[4]. S. G. Demos and R. R. Alfano, (1997) Optical polarization imaging, Appl. Opt. 36, 150-55.

[5]. M. Moscoso, J. B. Keller, and G. C. Papanicolaou, (2000) Depolarization and blurring of optical images by biological tissues. J. Opt. Soc. Am. A. 18, 948-60.

[6]. An sari MA, Massudi R, Hejazi M. Experimental and numerical study on simultaneous effects of scattering and absorption on fluorescence spectroscopy of a breast phantom. Opt Laser Techno 2009; 41(6): 74650 .

[7]. Vo-Dinh T. Biomedical Photonics Handbook. CRC Press; 2003.

[8]. Hecht E. Optics. Addison-Wesley; 2002.

[9]. Waynant RW. Lasers in Mediciene. Boca Raton: CRC Press; 2002.

[10]. Welch AJ, Gemert MVC. Optical-Response of Laser-Irradiated T issue. Springer; 1th rev; 1995.

[11] D. Hattery, V. Chernomordik, M. Loew, I. Gannot, and A. Gandjbakhche, "Analytical Solutions for Time-Resolved Fluorescence Lifetime Imaging in a Turbid Medium Such as Tissue," J. Opt. Soc. Am. A, vol. 18, no. 7, 2001, pp. 1523-1530.

[12] C. H. G. Wright, S. F. Barrett, and A. J. Welch, "Laser-Tissue Interaction," in Lasers in Medicine, D. R. Vij and K. Mahesh (eds. ), Kluwer, Boston, 2002. 